\title{
In Vivo Feasibility of Electrostatic Precipitation as an Adjunct to Pressurized Intraperitoneal Aerosol Chemotherapy (ePIPAC)
}

\author{
Tinatin Kakchekeeva, $\mathrm{MD}^{1}$, Cedric Demtröder, $\mathrm{MD}^{2}$, Nirmitha I. Herath, $\mathrm{PhD}^{3}$, Dominic Griffiths, $\mathrm{PhD}^{4}$, \\ Jared Torkington, $\mathrm{MD}^{5}$, Wiebke Solaß, $\mathrm{MD}^{6}$, Marie Dutreix, $\mathrm{PhD}^{7}$, and Marc A. Reymond, $\mathrm{MD}, \mathrm{MBA}^{1,2}$ \\ ${ }^{1}$ Department of Surgery, Otto-von-Guericke University Magdeburg, Magdeburg, Germany; ${ }^{2}$ Department of Surgery, \\ Marien Hospital Herne, Ruhr University Bochum, Herne, Germany; ${ }^{3}$ DNA Therapeutics, Évry, France; ${ }^{4}$ Alesi Surgical \\ Ltd., Cardiff, Wales, UK; ${ }^{5}$ Welsh Institute for Minimal Access Therapy (WIMAT), Cardiff, Wales, UK; ${ }^{6}$ Institute of \\ Pathology, Medical School Hanover, Hannover, Germany; ${ }^{7}$ Institut Curie, Orsay, France
}

\begin{abstract}
Background. Intraperitoneal chemotherapy is limited by tissue penetration. Pressurized intraperitoneal aerosol chemotherapy (PIPAC) has been shown to improve drug uptake by utilizing the physical properties of gas and pressure. This study investigated the effect of adding electrostatic precipitation to further enhance the pharmacologic properties of this technique.

Methods. A comparative study was performed using an in vivo porcine model. There were 3 cases in each group, PIPAC and electrostatic precipitation pressurized intraperitoneal aerosol chemotherapy (ePIPAC), plus 1 negative control comparing intraperitoneal distribution and tissue uptake of 2 tracer substances (toluidine blue and DT01). Tracer uptake was determined by measuring DT01 in tissue and peritoneal fluid at the end of each procedure. Results. Electrostatic precipitation of the aerosol was technically feasible in all ePIPAC animals. The aerosol was cleared completely from the visual field within $15 \mathrm{~s}$ in the ePIPAC group versus $30 \mathrm{~min}$ in the PIPAC group. The
\end{abstract}

Tinatin Kakchekeeva and Cedric Demtröder have contributed equally to this article, and both should be considered first author.

Electronic supplementary material The online version of this article (doi:10.1245/s10434-016-5108-4) contains supplementary material, which is available to authorized users.

(C) The Author(s) 2016. This article is published with open access at Springerlink.com

First Received: 14 September 2015;

Published Online: 2 February 2016

M. A. Reymond, MD, MBA

e-mail: marc.reymond@yahoo.de;

marc.reymond@marienhospital-herne.de peritoneal surface was homogeneously stained in both groups. After $30 \mathrm{~min}, 1.5 \%$ remaining DT01 was measured in samples of ePIPAC-treated peritoneal fluid versus $15 \%$ in PIPAC animals $(p=0.01)$. Tissue concentration was increased after ePIPAC versus PIPAC $(p=0.06)$.

Conclusions. ePIPAC is technically feasible and improves tissue uptake of 2 tracer substances compared to PIPAC by up to tenfold. Intraperitoneal distribution was homogeneous in both groups. ePIPAC has the potential to allow more efficient drug uptake, further dose reduction, a significant shortening of the time required for PIPAC application, and improved health and safety measures.

Research on tumor nonresponse to therapy has focused on the molecular mechanisms of chemoresistance, with drug distribution being comparatively neglected. ${ }^{1}$ For cytostatic drugs to be successful, they must fully penetrate the tissue of interest, reaching within all the cancer cells at a concentration sufficient to exert a therapeutic effect.

Intraperitoneal tumor dissemination and metastasis is common in several forms of abdominal cancer. ${ }^{2}$ Numerous studies have investigated the potential role of intraperitoneal drug delivery as an adjunct to systemic chemotherapy in this situation. ${ }^{3}$ The rationale of intraperitoneal administration is to improve the therapeutic index by increasing the exposure of cancer cells within the peritoneal cavity to the drug while minimizing toxic effects to other organs. Prior studies have documented the limitations of intraperitoneal chemotherapy including the limited direct penetration of drugs into the tumor tissue and the unequal drug distribution throughout the peritoneal cavity. ${ }^{4}$

Pressurized intraperitoneal aerosol chemotherapy (PIPAC) is an innovative drug delivery system that takes advantage of the physical properties of the combination of 
gas and pressure in order to overcome these pharmacologic limitations. ${ }^{5}$ There is substantial in vitro, in vivo, and ex vivo evidence as well as evidence in human patients that PIPAC has superior pharmacologic properties. ${ }^{6-9}$ Because the therapeutic ratio between local and systemic drug concentration is increased by PIPAC, enhanced local efficacy together with low systemic toxicity was expected and has been demonstrated clinically. ${ }^{10}$ Retrospective analysis of the patient cohorts in ovarian gastric and colorectal cancer have shown encouraging results of repeated PIPAC in the palliative situation. ${ }^{11-13}$ A prospective phase 2 trial with low-dose doxorubicin and cisplatin in recurrent, platin-resistant ovarian cancer applied as a pressurized aerosol showed a clinical benefit rate of $62 \%$ and an objective histologic regression rate of $76 \%$, coupled with a low incidence of severe adverse events $(15 \%$ Common Terminology Criteria for Adverse Events [CTCAE] grade 3, no CTCAE grades 4 or 5). ${ }^{14,15}$ So far, the role of PIPAC in combination with advanced cytoreductive surgery has not been determined.

We hypothesized that electrostatic precipitation may further enhance the pharmacologic properties of PIPAC as so-called electrostatic precipitation pressurized intraperitoneal aerosol chemotherapy (ePIPAC). For electrostatic precipitation, we used a commercially available, CE-certified technology developed for clearing surgical smoke from the operative field of view during laparoscopy (Ultravision, Alesi Surgical Ltd., UK). The performance and safety of Ultravision has been demonstrated in bench studies, preclinical testing, and clinical testing, including a clinical study on 30 patients undergoing laparoscopic cholecystectomy. ${ }^{16,17}$ In particular, no adverse events such as cardiac arrhythmia, modification of ECG, bowel perforations, or skin burning were reported.

The aims of this study were to assess the technical feasibility of ePIPAC, to compare the homogeneity of intraperitoneal distribution between PIPAC and ePIPAC, and to determine possible improvement of tissue uptake after ePIPAC.

\section{MATERIALS AND METHODS}

Study Design

This was an exploratory experimental in vivo study in a large animal model comparing the effect of ePIPAC (3 animals) versus PIPAC ( 3 animals) versus 1 control animal (ePIPAC, no stain).

\section{Animal Model}

The experiment was performed in compliance with the German Animal Protection Law (TierSchG 2006) and was authorized by the competent authority, State of Thuringia. ARRIVE guidelines were implemented. Seven German nonsyngeneic landrace pigs (5 females, 2 males) weighing $31.5 \pm 4.5 \mathrm{~kg}$ were operated on by qualified surgeons under the supervision of a veterinarian. The sample size was determined in order to obtain experimental results in triplicate plus a negative control. Animals were randomly assigned to the experimental groups. Procedures were performed under general anesthesia adhering to strict protocols. The animals were euthanized under narcosis at the end of the procedure and immediately necropsied. The primary outcome measure was DT01 concentration in tissue and peritoneal fluid at the end of the procedures (quantitative measurement). Secondary outcomes were the homogeneity of blue staining within the peritoneal cavity (qualitative measurement) and the toluidine blue concentration in the peritoneal fluid at the end of the procedure (semiquantitative measurement).

\section{Staining Substances}

DT01 are noncoding small DNA fragments designed to bait and hijack the enzyme complexes that repair DNA double-strand breaks, diverting them away from their primary objective, the double-strand breaks on chromosomes. ${ }^{15,18}$ DT01 administration with PIPAC has previously been validated. ${ }^{8}$ In this study, $30 \mathrm{mg}$ toluidine blue and $6 \mathrm{mg}$ Cy5-labeled DT01 were diluted in $1000 \mathrm{ml}$ $\mathrm{NaCl} 0.9 \%$ solution. A volume of $150 \mathrm{ml}$ solution was administered via an aerosolizer (Capnopen, Capnomed $\mathrm{GmbH}$, Germany) to each animal $(n=6)$. The negative control animal received $150 \mathrm{ml} \mathrm{NaCl} 0.9 \%$.

\section{Experimental Protocol}

PIPAC was applied as described previously. ${ }^{9}$ After insufflation of a $12 \mathrm{~mm} \mathrm{Hg}$ capnoperitoneum with a Veress needle, 2 balloon safety trocars (Kii 5 and $12 \mathrm{~mm}$; Applied Medical, Germany) were inserted into the abdominal wall. The Capnopen was connected to an intravenous highpressure injector (Arterion 7; Medrad, Germany) and inserted into the abdomen. The pressurized aerosol was applied via aerosolizer and injector. Flow rate was $30 \mathrm{ml} /$ min, and maximal upstream pressure was 200 psi. The therapeutic capnoperitoneum was maintained for $30 \mathrm{~min}$. The aerosol was exsufflated and the trocars removed. Identical conditions were used for the ePIPAC subjects $(n=3)$, with the additional use of the Ultravision technology. The system was activated at the point of completion of aerosol generation and the electric current was maintained for $30 \mathrm{~min}$. This negative control animal received $\mathrm{NaCl} 0.9 \%$ through ePIPAC under the above conditions. 


\section{Electrostatic Precipitation}

The Ultravision system integrates the following components: a generator unit (voltage 7500-9500 V, current $\leq 10 \mu \mathrm{A}$ ), an active cable terminating in an atraumatic stainless steel brush electrode (Ionwand) that is responsible for the electrostatic charging of aerosol particles, and a return electrode with a solid patient return plate (Fig. 1). The Ionwand emits a stream of electrons, resulting in the creation of negative gas ions. The gas ions collide with particulate matter, passing on the negative charge. The return electrode confers a weak positive charge on the subject, which results in the electrostatic attraction of the negatively charged aerosol particles to the tissue surfaces of the contained space-that is, the peritoneum.

\section{Analysis}

Toluidine blue distribution was assessed qualitatively as described previously. ${ }^{7}$ Immediately after the procedure, peritoneum was sampled via biopsy; peritoneal fluid was sampled, and droplets were distributed onto filter paper to visualize the intensity of the blue stain. The tissue and peritoneal fluid samples were snap-frozen in liquid nitrogen and processed at the Institut Curie/Orsay for blinded analysis. The quantitation of DT01 in the peritoneal fluid was performed by a hybridization enzyme-linked immunosorbent assay (ELISA) using a biotin-conjugated capture oligonucleotide probe $(300 \mu \mathrm{l})$ and a digoxigeninconjugated detection probe $(300 \mu \mathrm{l})$, with sequences complementary to the DT01 sequence (Exiqon, USA) in 96-well plates. Then samples were incubated with an antidigoxigenin horseradish peroxidase-conjugated antibody (1:10,000; Roche, USA), and detection was performed by the addition of $3,3^{\prime}, 5,5^{\prime}$-tetramethylbenzidine substrate $(100 \mu \mathrm{l})$. Absorbance was measured at 450 and $560 \mathrm{~nm}$, and quantity of DT01 was calculated from calibration standards over a working range of $25-1000 \mathrm{ng} / \mathrm{ml}$ using a 4-parameter logistic curve. Because the ELISA failed to produce reliable quantification in tissues, we used fluorescent quantification, a reliable technique for assessing molecule distribution. ${ }^{19}$ Peritoneal tissue samples were defrosted, then crushed in phosphate-buffered salineethylenediaminetetraacetic acid. Fifty microliters of each extract was formed into aliquots in a 96-well plate, and fluorescence was measured with a Typhoon scanner (GE Healthcare, USA). The quantity of DT01 was calculated from a standard curve performed using peritoneal extract from untreated groups, with Cy5-labeled DT01 concentrations ranging from 0 to $1 \mu \mathrm{g} / \mathrm{ml}$.

\section{Statistics}

Size sample was determined and limited by the decision of the regulatory authority: experiments in triplicate $(2 \times 3)$, plus 1 control, for a total of 7 animals. Descriptive

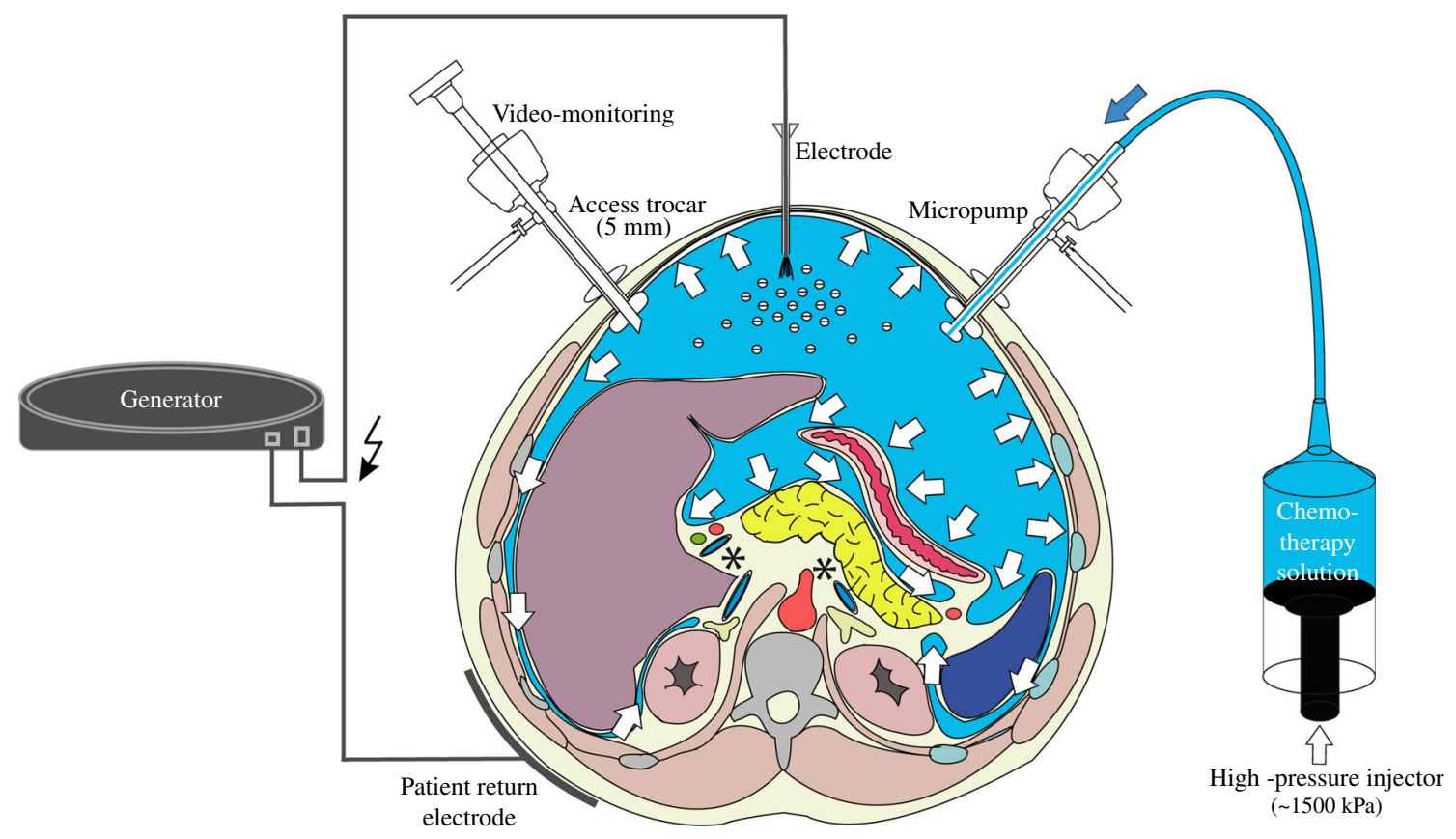

FIG. 1 Principle of electrostatic precipitation ePIPAC. a Technical setting for ePIPAC, including high-pressure injector containing therapeutic solution micropump generating pressurized intraperitoneal aerosol, brush electrode for electrostatic loading of therapeutic aerosol, and return electrode (solid plate). b Intraoperative view of abdomen showing micropump producing aerosol and electrode actively loading this aerosol with electrostatic charges, leading to precipitation of aerosol particles 
statistics including mean and standard deviation are provided. The null hypothesis was that DT01 concentration was equal in the peritoneal fluid and in peritoneal biopsy samples of PIPAC and ePIPAC animals. In spite of the small sample size, an exploratory comparative analysis was performed by a nonparametric test (Kruskal-Wallis test for independent samples). A $p$ value of $<0.05$ was considered significant.

\section{RESULTS}

There were no technical difficulties or intraoperative complications in any of the cases. In both the PIPAC and ePIPAC groups, rapid nebulization of the toluidine blue solution within the tightly closed abdomen was observed. Videoscopic control showed immediate staining of the complete abdominal cavity in both PIPAC and ePIPAC animals, including all exposed peritoneal surfaces. Intraabdominal organs were not mobilized.

In the ePIPAC group, electrostatic loading of the saline aerosol was technically feasible without significant aberrant conduction. The maximal allowed current intensity was not reached in the ePIPAC group, denoted by the absence of an alarm signal. After activation of the electrode in the ePIPAC group, the aerosol completely cleared from the field of view within $15 \mathrm{~s}$, as documented by real-time videoendoscopy. In contrast, in the PIPAC animals, aerosol particles remained in suspension until the end of the procedure (after 30 min of steady state).

At necropsy, macroscopic stain distribution throughout the entire peritoneal cavity was homogeneous in the PIPAC group, including the small bowel and anterior abdominal wall and hidden surfaces such as the inferior aspect of the liver and the liver hilum. Comparable results were obtained in the ePIPAC group. In particular, no staining gradient toward or from the brush electrode was observed in the ePIPAC animals (Fig. 2). No bowel lesion or perforation was noted.

After $30 \mathrm{~min}$, peritoneal fluid still demonstrated the blue staining in the PIPAC group (Supplementary material 1, arrows), whereas it was greatly reduced in the ePIPAC group. This qualitative impression was confirmed by the absence of color on filter paper with peritoneal fluid after ePIPAC. In contrast, blue staining of the peritoneal fluid was still present after PIPAC.

The concentration of Cy5-labeled DT01 in the peritoneal fluid was lower after ePIPAC compared to PIPAC (Fig. 3a), confirming the results obtained from the toluidine blue assessment. The mean initial DT01 concentration in the aerosolized solution was $8.93 \pm 0.72 \mu \mathrm{g} / \mathrm{ml}$. After PIPAC, this concentration
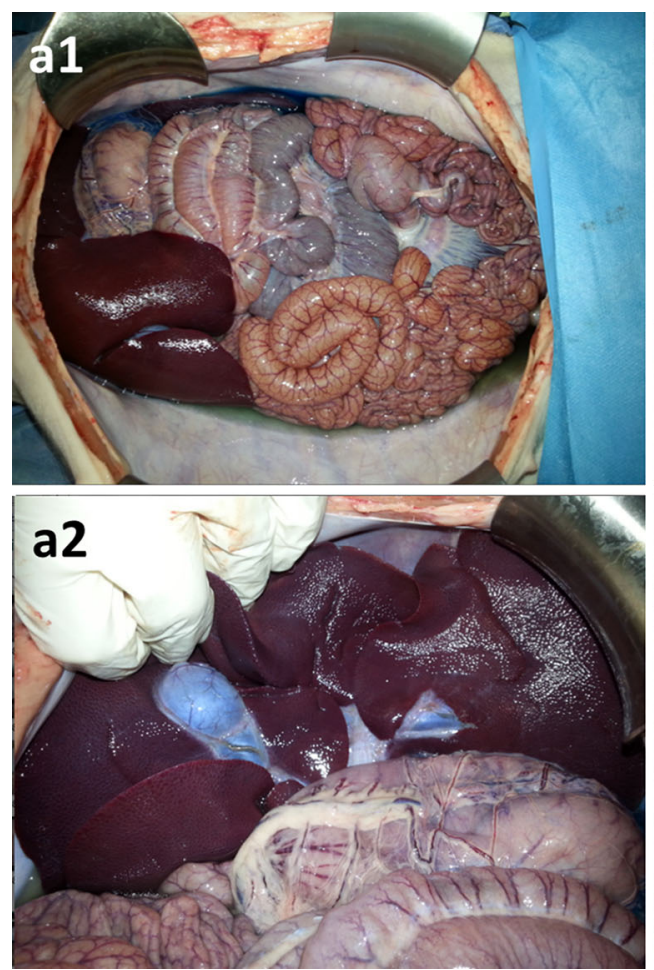

FIG. 2 Adequacy of toluidine blue distribution. Autopsy findings in $\operatorname{PIPAC}(\mathbf{a 1}, \mathbf{a 2})$ and ePIPAC (b1, b2) animals after aerosolization of low-dose toluidine blue. Staining of serosal surfaces is homogeneous
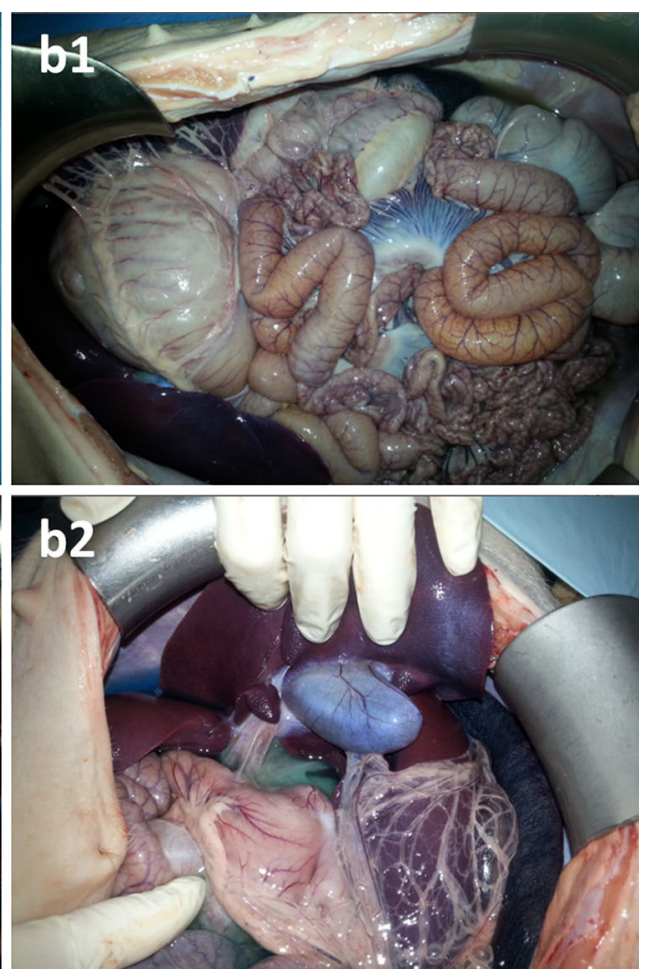

in both groups. Importantly, inferior aspect of liver, including hilum and gallbladder, are stained 

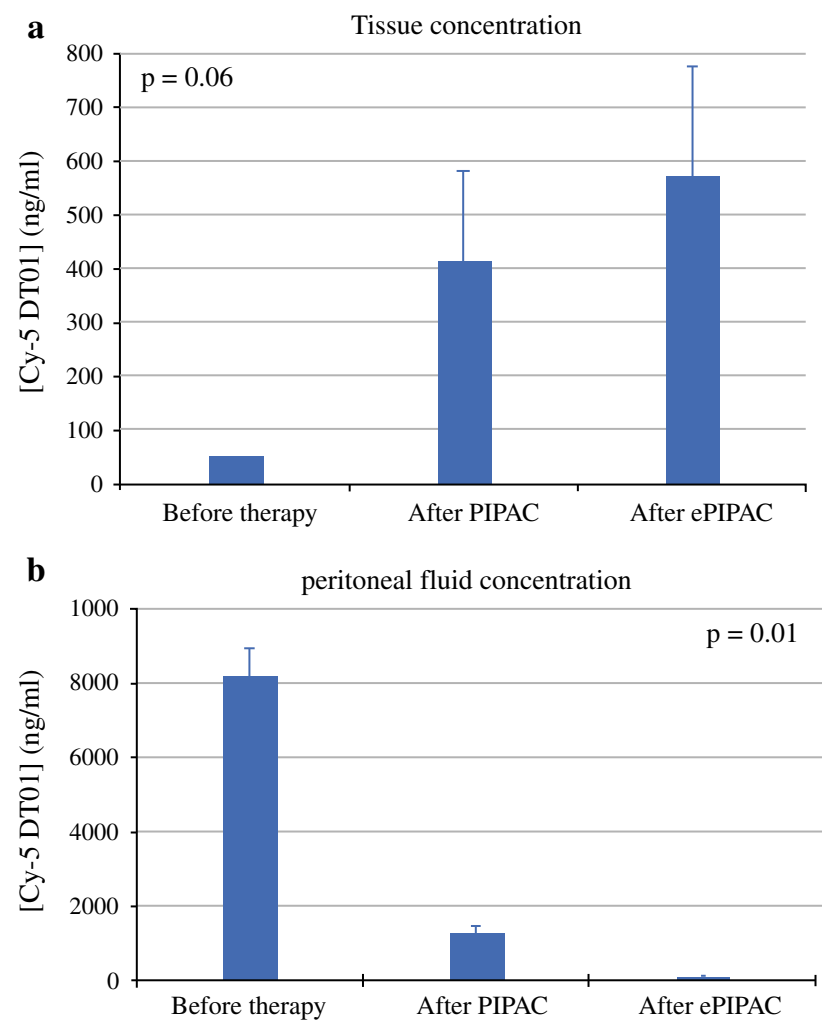

FIG. 3 a Peritoneal fluid DT01 concentration showing $15 \%$ remaining concentration after PIPAC compared to initial concentration in aerosolized solution versus $1.5 \%$ after ePIPAC $(p=0.01)$. Whereas PIPAC allows $85 \%$ tissue uptake, ePIPAC achieves another order of magnitude with $98.5 \%$ absorption. b Tissue DT01 concentration after PIPAC vs. ePIPAC application, confirming superior uptake after ePIPAC $(p=0.06)$

diminished to $1.46 \pm 0.21 \mu \mathrm{g} / \mathrm{ml}$, indirectly documenting a tissue uptake of $83.6 \%$ in this closed system. After ePIPAC, the concentration further dropped to $0.11 \pm 0.02 \mu \mathrm{g} / \mathrm{ml}$, suggesting an almost complete tissue uptake of DT01 $(98.7 \%)$. The null hypothesis could therefore be rejected $(p=0.01)$. Superior DT01 uptake after ePIPAC versus PIPAC was confirmed by tissue measurement (Fig. 3b): DT01 concentration in tissue increased from $0.05 \mu \mathrm{g} / \mathrm{ml}$ (background noise) before therapy to $0.41 \pm 0.17 \mu \mathrm{g} / \mathrm{ml}$ after PIPAC application and to $0.57 \pm 0.20 \mu \mathrm{g} / \mathrm{ml}$ after ePIPAC application $(p=0.06)$.

\section{DISCUSSION}

In this experimental study, we combined 3 physical principles (electrostatic precipitation, aerosol nature, and hydrostatic pressure) with the aim of further improving tissue uptake after intraperitoneal delivery, thus developing the concept of ePIPAC.

Electrostatic loading of a therapeutic aerosol was technically feasible in all ePIPAC animals in an environment highly saturated with saline solution without creating significant erratic electric currents. The aerosol was cleared much more quickly in ePIPAC animals compared to PIPAC animals. No major macroscopic differences were noted in dye distribution; in particular, there was no optical tissue staining gradient toward or from the active electrode in the ePIPAC group. At the end of the procedure, the peritoneal fluid was colorless in the ePIPAC group while the blue color was maintained in the PIPAC group, suggesting a more effective clearance of toluidine blue from the aerosol after electrostatic precipitation. Subsequent semiquantitative analysis with filter paper confirmed this clinical impression. Quantitative results obtained with the second tracer (DT01) demonstrated that transfer of the tracer from the aqueous solution to tissue surfaces was improved after ePIPAC compared to PIPAC. After PIPAC, approximately $1 / 10$ of the DT01 was present within the peritoneal cavity, while with ePIPAC only about $1 / 10^{2}$ remained. DT01 is a much larger molecule than toluidine blue, and therefore such efficient uptake was not anticipated. This superior uptake after ePIPAC was confirmed by a higher tissue concentration than after PIPAC.

This difference in uptake represents a further improvement in the context of an earlier study in which tissue concentration of doxorubicin after PIPAC was found to be up to 200 times higher than reported after hyperthermic intraperitoneal chemotherapy (HIPEC), with only $10 \%$ of the dose. ${ }^{9}$

Application of intraperitoneal chemotherapy with ePIPAC may have several potential advantages over existing techniques:

- First, if it can be shown that increased deposition efficiency translates to an increased tissue uptake, then it may increase drug uptake into tumor nodes and therefore achieve cytotoxic dose in larger nodules. This could in turn reduce the need for aggressive cytoreductive surgery and allow therapy of diffuse small bowel involvement, a contraindication for cytoreductive surgery and HIPEC. ${ }^{20}$

- Second, it may allow a further reduction of the dose needed to be effective. In ovarian and gastric cancer, PIPAC has been shown to be effective with $10 \%$ of the usual systemic dose of cisplatin and doxorubicin. ${ }^{12,14}$ In colorectal cancer, PIPAC was effective with $20 \%$ of the doses generally administered for HIPEC. ${ }^{13}$ Dose reduction allows not only a reduction in organ toxicity and systemic side effects but also a limit to local toxicity on the bowel and the normal peritoneum. ${ }^{10,14}$ The clinical significance of this is the possibility to use PIPAC earlier in the course of the disease as a secondary prevention of peritoneal metastasis, analogous to HIPEC, and may contribute to a lower incidence of peritoneal sclerosis. ${ }^{21-25}$ 
- Third, ePIPAC may allow a significant reduction of the time needed for application.

- Finally, it may simplify the occupational safety aspects of PIPAC by reducing time of potential exposure and by minimizing any residual drug evacuated at the end of the procedure.

There are several limitations to this early work. These data have been obtained in an experimental model and cannot be extrapolated to human patients without further validation. This study was not performed in an animal model of peritoneal metastasis but in healthy pigs because such a model is not available. Therefore, tracer penetration into tumor tissue could not be assessed.

It was not possible to apply toxic agents such as cytotoxic drugs within the experimental operating room as a result of the absence of high-flow ventilation. Although DT01 is a validated marker for determining tissue drug uptake, the results presented are only valid for the substances tested. $^{8,26}$ It is likely that the ability to improve tissue deposition of drug substances using electrostatic precipitation will be affected by the physical characteristics of these molecules. Thus, it is not possible to extrapolate the clearance of toluidine blue and DT01 to the clearance of chemotherapy drugs. Additional pharmacologic studies are required for each drug used for ePIPAC.

\section{CONCLUSIONS}

The therapeutic effect of ePIPAC is through a combination of aerosolization of the drug, applying a pressure across it and application of an electrostatic gradient. ePIPAC is technically feasible and improves tissue uptake of 2 tracer substances compared to PIPAC. ePIPAC has the potential to allow more efficient drug uptake, to permit further dose reduction, to significantly shorten the time required for PIPAC application, and to improve health and safety in the operating room when undertaking such procedures.

ACKNOWLEDGMENT Supported in part by institutional funds and by an educational Grant and provision of the Ultravision system by Alesi Surgical Ltd. The authors thank Frank Pölzing, MD, for animal care, and Céline Agrario and Aurélie Herbette for technical assistance.

DISCLOSURE MAR has an equity interest in CAPNOMED $\mathrm{GmbH}$, Villingendorf, Germany. JT is a medical advisor and shareholder of Alesi Surgical Ltd., Cardiff, UK. DG is an employee of Alesi Surgical Ltd. CD received research funding from CAPNOMED. $\mathrm{NH}$ is an employee of DNA Therapeutics, Evry, France. MD holds an equity interest in DNA Therapeutics. The other authors declare no conflicts of interest.

OPEN ACCESS This article is distributed under the terms of the Creative Commons Attribution 4.0 International License (http:// creativecommons.org/licenses/by/4.0/), which permits unrestricted use, distribution, and reproduction in any medium, provided you give appropriate credit to the original author(s) and the source, provide a link to the Creative Commons license, and indicate if changes were made.

\section{REFERENCES}

1. Minchinton AI, Tannock IF. Drug penetration in solid tumors (review). Nat Rev Cancer. 2006;6:583-92.

2. Steinert R, Hantschick M, Vieth M, et al. Influence of subclinical tumor spreading on survival after curative surgery for colorectal cancer. Arch Surg. 2008;143:122-8.

3. Ceelen WP, Flessner MF. Intraperitoneal therapy for peritoneal tumors: biophysics and clinical evidence. Nat Rev Clin Oncol. 2010;7:108-15.

4. Dedrick RL, Flessner MF. Pharmacokinetic problems in peritoneal drug administration: tissue penetration and surface exposure. J Natl Cancer Inst. 1997;89:480-7.

5. Reymond MA, Hu B, Garcia A, et al. Feasibility of therapeutic pneumoperitoneum in a large animal model using a microvaporisator. Surg Endosc. 2000;14:51-5.

6. Haidira A, Pocard M. Nouveau mode d'administration en intrapéritonéal d'une chimiothérapie dans le traitement de la carcinose péritonéale: PIPAC (pressurized intraperitoneal aerosol chemotherapy). Master's thesis, University of Paris, 2015.

7. Solaß W, Hetzel A, Nadiradze G, et al. Description of a novel approach for intraperitoneal drug delivery and the related device. Surg Endosc. 2012;26:1849-55.

8. Solass W, Herbette A, Schwarz T, et al. Therapeutic approach of human peritoneal carcinomatosis with Dbait in combination with capnoperitoneum: proof of concept. Surg Endosc. 2012;26:84752.

9. Solass W, Kerb R, Mürdter T, et al. Intraperitoneal chemotherapy of peritoneal carcinomatosis using pressurized aerosol as an alternative to liquid solution: first evidence for efficacy. Ann Surg Oncol. 2014;21:553-9.

10. Blanco A, Giger-Pabst U, Solass W, Zieren J, Reymond MA. Renal and hepatic toxicities after pressurized intraperitoneal aerosol chemotherapy (PIPAC). Ann Surg Oncol. 2013;20:23116.

11. Tempfer CB, Celik I, Solass W, et al. Activity of pressurized intraperitoneal aerosol chemotherapy (PIPAC) with cisplatin and doxorubicin in women with recurrent, platinum-resistant ovarian cancer: preliminary clinical experience. Gynecol Oncol. 2014;132:307-11.

12. Nadiradze G, Giger-Pabst U, Zieren J, Strumberg D, Solass W, Reymond MA. Pressurized intraperitoneal aerosol chemotherapy (PIPAC) with low-dose cisplatin and doxorubicin in gastric peritoneal metastasis. J Gastrointest Surg. 2016;20:367-73.

13. Demtröder C, Solass W, Zieren J, Strumberg D, Giger-Pabst U, Reymond MA. Pressurized intraperitoneal aerosol chemotherapy (PIPAC) with oxaliplatin in colorectal peritoneal metastasis. Colorectal Dis. 2015. doi:10.1111/codi.13130.

14. Tempfer CB, Winnekendonk G, Horvat R, et al. Pressurized intraperitoneal aerosol chemotherapy (PIPAC) with cisplatin and doxorubicin in women with recurrent, platinum-resistant ovarian cancer: an open-label, single-arm, phase 2 study (PIPAC-OV1). Gynaecol Oncol. 2015;137:223-8.

15. Croset A, Cordelières FP, Berthault N, et al. Inhibition of DNA damage repair by artificial activation of PARP with siDNA. Nucleic Acids Res. 2013;41:7344-55.

16. Ansell J, Warren N, Wall $\mathrm{P}$, et al. Electrostatic precipitation is a novel way of maintaining visual field clarity during laparoscopic surgery: a prospective double-blind randomized controlled pilot study. Surg Endosc. 2014;28:2057-65. 
17. Alesi Surgical, Cardiff, UK. Available at: http://www.alesisurgical.com/. Accessed April 29, 2015.

18. Helleday T, Petermann E, Lundin C, Hodgson B, Sharma RA. DNA repair pathways as targets for cancer therapy. Nat Rev Cancer. 2008;8:193-204.

19. Vasquez KO, Casavant C, Peterson JD. Quantitative whole body biodistribution of fluorescent-labeled agents by non-invasive tomographic imaging. PLoS One. 2011;6:e20594.

20. Elias D, Mariani A, Cloutier AS, et al. Modified selection criteria for complete cytoreductive surgery plus HIPEC based on peritoneal cancer index and small bowel involvement for peritoneal carcinomatosis of colorectal origin. Eur J Surg Oncol. 2014;40: 1467-73.

21. Elias D, Goéré D, Dumont F, et al. Role of hyperthermic intraoperative peritoneal chemotherapy in the management of peritoneal metastases. Eur J Cancer. 2014;50:332-40.

22. Takebayashi K, Sonoda H, Shimizu T, et al. Successful surgical approach for a patient with encapsulating peritoneal sclerosis after hyperthermic intraperitoneal chemotherapy: a case report and literature review. BMC Surg. 2014;14:57.
23. Chan WS, Bohmer R, McIntosh R, Blomfield P. Peritoneal fibrosis leading to small bowel obstruction two years after firstline intraperitoneal chemotherapy for optimally debulked ovarian cancer. Aust N Z J Obstet Gynaecol. 2011;51:91-2.

24. Aihara H, Maruoka H, Kiyozaki H, Konishi F. Sclerosing encapsulating peritonitis (SEP) as a delayed complication of continuous hyperthermic peritoneal perfusion (CHPP): report of a case. Surg Today. 2003;33:232-6.

25. Vlasveld LT, Taal BG, Kroon BB, Gallee MP, Rodenhuis S. Intestinal obstruction due to diffuse peritoneal fibrosis at 2 years after the successful treatment of malignant peritoneal mesothelioma with intraperitoneal mitoxantrone. Cancer Chemother Pharmacol. 1992;29:405-8.

26. Wagner P, Boone B, Ramalingam L, et al. Histologic and immunohistochemical alterations associated with cytoreductive surgery and heated intraperitoneal chemotherapy. Ann Surg Oncol. 2015; 22(Suppl 3):S588-95. 